\title{
A new tool for touch-free patient registration for robot-assisted intracranial surgery: application accuracy from a phantom study and a retrospective surgical series
}

\author{
Francesco Cardinale, MD, PhD, ${ }^{1}$ Michele Rizzi, MD, ${ }^{1,2}$ Piergiorgio d'Orio, MD, ${ }^{1}$ \\ Giuseppe Casaceli, MD, ${ }^{3}$ Gabriele Arnulfo, PhD, ${ }^{4}$ Massimo Narizzano, $\mathrm{PhD},{ }^{4}$ \\ Davide Scorza, MSc ${ }^{5,6}$ Elena De Momi, PhD, ${ }^{5}$ Michele Nichelatti, PhD, ${ }^{7}$ Daniela Redaelli, MD, ${ }^{8}$ \\ Maurizio Sberna, MD, ${ }^{8}$ Alessio Moscato, MSc, ${ }^{9}$ and Laura Castana, MD $^{1}$ \\ 1"Claudio Munari” Center for Epilepsy Surgery and Departments of 'Biostatistics and ${ }^{8}$ Neuroradiology, Niguarda Hospital; \\ ${ }^{3}$ Department of Neurosurgery, San Carlo Borromeo Hospital; ${ }^{5}$ Department of Electronics, Information, and Bioengineering, \\ Politecnico di Milano; ${ }^{9}$ Department of Medical Physics, Bassini Hospital-Cinisello Balsamo, Milan; ${ }^{2}$ Department of Neuroscience, \\ University of Parma; ${ }^{4}$ Department of Informatics, Bioengineering, Robotics, and System Engineering (DIBRIS), University of \\ Genova, Italy; and ${ }^{6}$ eHealth and Biomedical Applications, Vicomtech-IK4, San Sebastián, Spain
}

\begin{abstract}
OBJECTIVE The purpose of this study was to compare the accuracy of Neurolocate frameless registration system and frame-based registration for robotic stereoelectroencephalography (SEEG).

METHODS The authors performed a 40-trajectory phantom laboratory study and a 127-trajectory retrospective analysis of a surgical series. The laboratory study was aimed at testing the noninferiority of the Neurolocate system. The analysis of the surgical series compared Neurolocate-based SEEG implantations with a frame-based historical control group.

RESULTS The mean localization errors (LE) \pm standard deviations (SD) for Neurolocate-based and frame-based trajectories were $0.67 \pm 0.29 \mathrm{~mm}$ and $0.76 \pm 0.34 \mathrm{~mm}$, respectively, in the phantom study $(p=0.35)$. The median entry point LE was $0.59 \mathrm{~mm}$ (interquartile range [IQR] $0.25-0.88 \mathrm{~mm}$ ) for Neurolocate-registration-based trajectories and $0.78 \mathrm{~mm}$ (IQR $0.49-1.08 \mathrm{~mm}$ ) for frame-registration-based trajectories $(p=0.00002)$ in the clinical study. The median target point LE was $1.49 \mathrm{~mm}$ (IQR 1.06-2.4 mm) for Neurolocate-registration-based trajectories and $1.77 \mathrm{~mm}$ (IQR 1.25-2.5 mm) for frameregistration-based trajectories in the clinical study. All the surgical procedures were successful and uneventful.

CONCLUSIONS The results of the phantom study demonstrate the noninferiority of Neurolocate frameless registration. The results of the retrospective surgical series analysis suggest that Neurolocate-based procedures can be more accurate than the frame-based ones. The safety profile of Neurolocate-based registration should be similar to that of frame-based registration. The Neurolocate system is comfortable, noninvasive, easy to use, and potentially faster than other registration devices.
\end{abstract}

https://thejns.org/doi/abs/10.3171/2017.2.FOCUS16539

KEY WORDS SEEG; robotics; Neuromate; Neurolocate; frameless; epilepsy surgery; depth electrodes; image-guided surgery

$\mathrm{S}$ URGERY is an effective option for the treatment of drugresistant epilepsy ${ }^{28}$ for patients in whom the epileptogenic zone, defined as "the site of the beginning and of primary organization of the epileptic seizures," ${ }^{21}$ can be safely removed. Despite advancements in the presurgical noninvasive workup, a number of patients still undergo intracranial electroencephalography. ${ }^{13,16,17,19,21,22}$ Stereoelec- troencephalography (SEEG) is one of the most prominent methods for direct recording of brain electrical activity and has recently spread beyond the European borders.., 24 This method is safe, when performed according to rigorous principles. ${ }^{3,20}$ Many stereotactic devices can be used for the implantation of intracerebral electrodes for SEEG. The use of a robotic assistant has recently gained popular-

ABBREVIATIONS FM = fiducial marker; IQR = interquartile range; $L E=$ localization error; SEEG = stereoelectroencephalography; US = ultrasound.

SUBMITTED December 17, 2016. ACCEPTED February 20, 2017.

INCLUDE WHEN CITING DOI: 10.3171/2017.2.FOCUS16539. 
ity, with excellent results in terms of application accuracy and safety. ${ }^{1,11,12,15,25}$ Patient registration can be performed in frame-based or frameless conditions. Since the fall of 2008, we have used a robotic assistant in frame-based conditions for implanting all SEEG electrodes at Niguarda Hospital. ${ }^{4}$ A new device for frameless registration, compatible with our robot, has recently become available. The purpose of this paper is to describe the results of 2 studies aimed at comparing the accuracy provided by this new device with that of frame-based procedures.

\section{Methods}

The present article reports on the results of 2 independent studies. The first is a laboratory study performed on a phantom; the second is a retrospective analysis of a convenience surgical series. Both studies were aimed at assessing the stereotactic accuracy of robot-assisted SEEG electrode implantation using the Neurolocate patient registration module (Renishaw Mayfield SA).

The clinical use of the robotic assistant in frame-based conditions at our center was detailed in previously published papers. ${ }^{4,6,7}$

\section{The Neurolocate Device}

The Neurolocate device is a new, frameless patient registration module designed to be used with the Neuromate stereotactic robot (Renishaw Mayfield), a 5-degreesof-freedom passive robot. The Neurolocate module has 5 synthetic ruby balls (Neurolocate fiducial markers [FMs]), which are attached to an FM frame by means of 5 carbonfiber rods (Fig. 1). The FM frame is, in turn, attached to the laser tool holder, which is mounted on the Neuromate arm.

The robotic arm is first moved so that the Neurolocate module is very close to the head of the patient, after the

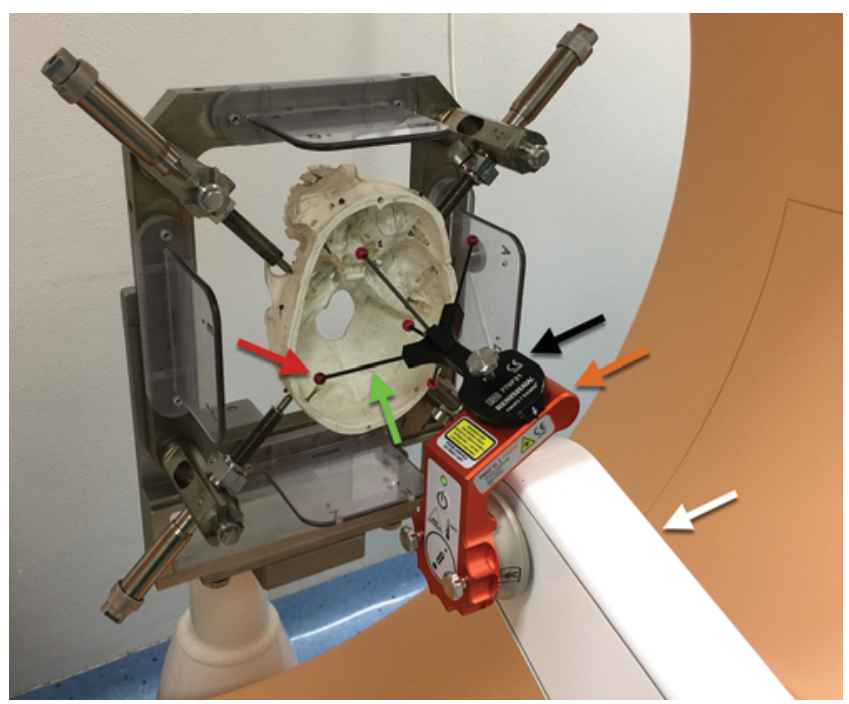

FIG. 1. Photograph of the phantom study setup with the Neurolocate frameless registration module in place. The Neurolocate module is made of a body (black arrow) with 5 carbon-fiber rods (green arrow) supporting synthetic ruby balls (red arrow). The module is attached to the robotic arm (white arrow) by means of the laser holder (orange arrow). head holder is attached to the robot, at the beginning of the patient registration.

A 3D data set is then obtained with an intraoperative CT scanner. Finally, a specific software module is used to complete the registration, selecting the center of the Neurolocate FMs in the multiplanar reconstructions provided by the Voxim stereotactic planning software (IVS Technology $\mathrm{GmbH}$ ) (Fig. 2). The planning software can compute the transformation matrix from the image space to the robot space, because the positions of the 5 Neuromate FMs are sent by the robot to the control workstation. Thus, once the registration has been completed, the robotic arm
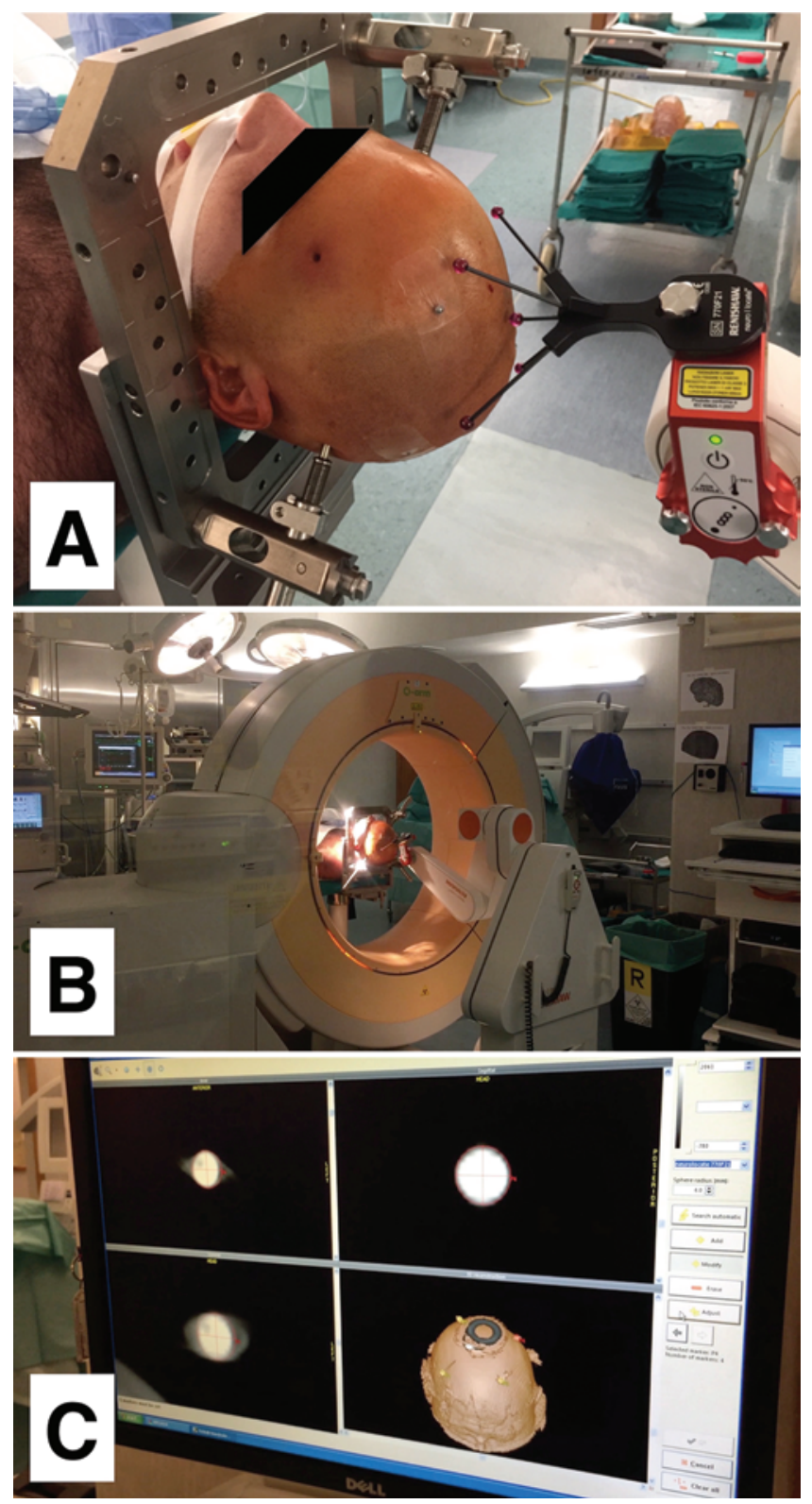

FIG. 2. Basic steps of Neurolocate registration. A: The Neurolocate module is brought very close to the patient's head, without touching it, by moving the robotic arm with a remote control. B: A 3D image data set is obtained with the $\mathrm{O}$-arm. C: The centers of the Neurolocate FMs are selected in multiplanar reconstructions in a semiautomated procedure by means of the stereotactic software. 
can align the tool holder with the vector of the planned trajectories.

\section{Cone-Beam CT}

For both the phantom study and the clinical case series, imaging data sets were acquired with an O-arm 1000 system (Medtronic). This cone-beam CT scanner is able to produce 3D CT-like data sets and 2D projective x-ray images. The reconstructed 3D volume is a $200 \times 150-\mathrm{mm}$ cylinder, described by a series of 12-bit DICOM (Digital Imaging and Communications in Medicine) files (192 axial slices, $512 \times 512$ matrix, $0.415 \times 0.415 \times 0.833-\mathrm{mm}$ anisotropic voxels). We acquired data sets using the preset high-definition and the standard-definition protocols, respectively, for the phantom study and the clinical retrospective study.

\section{Phantom Study}

This was a noninferiority study aimed at testing the null hypothesis that the stereotactic accuracy of Neurolocate-based frameless procedures does not differ from that of Talairach-frame-based procedures.

The phantom was a humanlike plastic skull without the cranial vault. Ten internal FMs (Cranial Marker System, Leibinger) were fixed to the skull, covering the 3 cranial fossae, bilaterally. Each phantom FM is made of a cranial screw supporting a removable CT-visible target. The phantom was fixed to the pillars of a Talairach stereotactic frame by means of 4 conical pins. The frame was attached to the Neuromate robotic assistant by means of a dedicated support (Fig. 3).

The measuring probe supported a synthetic ruby ball as an FM on its tip (probe FM), easily visible on our CT-like data sets. The probe was fixed to the tool holder of the robotic arm by means of a dedicated adapter with the length set at $100 \mathrm{~mm}$ (Fig. 4).

Two experiments (Experiments 1 and 2) were performed. In both experiments registration was performed in a frameless condition with the Neurolocate module and

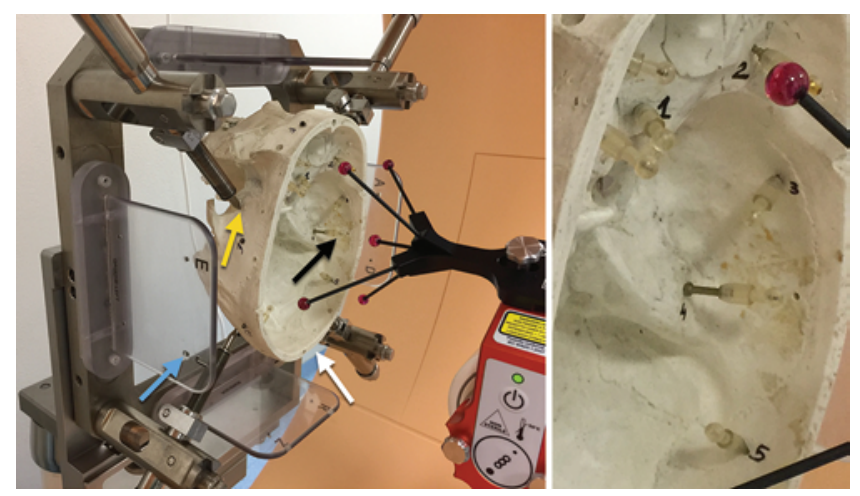

FIG. 3. Left: Photograph of the phantom study setup showing the attachment of the plastic skull to the Talairach frame, the FMs, and the localizers. The plastic skull (white arrow) is attached to the pillars of the Talairach frame by means of 4 conical pins (yellow arrow). Ten FMs with removable targets (black arrow) are fixed to the internal skull. The localizers (blue arrow) for bidimensional x-ray registration are attached to the base of the frame. Right: Close-up view of the plastic skull and removable targets.

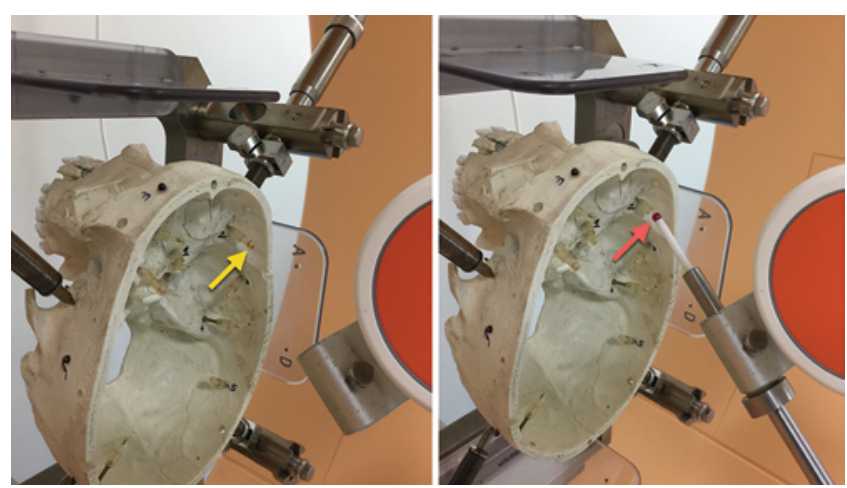

FIG. 4. Photographs illustrating the FM targeting in the phantom study. Left: The target of FM 2 is indicated by the yellow arrow. Right: The measuring probe is driven to the target (red arrow pointing to the tip of the probe), once the fiducial marker is removed.

in a frame-based condition with the dedicated x-ray localizers. The position of the phantom was changed between the 2 experiments, simulating 2 different surgeries. The experiments were otherwise identical.

Neurolocate was brought close to the phantom, at the beginning of each experiment. A 3D planning data set was obtained, as long as 2 bidimensional (2D) radiographs in anteroposterior and laterolateral projection. The Neurolocate registration was then completed with Voxim (Fig. 3). Of note, the Tailarach frame served only as a head holder, when the Neurolocate module was used. Ten trajectories were then planned, targeting the 10 phantom FMs. The phantom FM target was removed from its support, and the probe was advanced, ideally placing the center of the probe FM in the same position of the removed FM target. This was performed for each trajectory, obtaining 10 image data sets. The frame-based part of the experiment started after the Neurolocate part of the experiment was completed. The 2 radiographs previously obtained with the planning data set were uploaded into Voxim, and the dedicated localizers were selected in both projections, as in the clinical setup previously described. ${ }^{4,6}$ A point-topoint registration was subsequently performed between the $2 \mathrm{D}$ images and the 3D data set, selecting the Neurolocate FMs and some of the phantom FMs on both the image modalities. Thus, the phantom position inside the frame space was registered to the robotic workspace. Next, 10 image data sets were obtained for visualizing the position of the probe FM, targeting the 10 phantom FMs, as in the previous section of the experiment.

Overall, 40 trajectories were planned (20 in each experiment), and 40 image data sets were acquired with the measurement probe in position. Each data set with the positioned probe FM was automatically registered to the planning data set, and the quality of the registration was visually checked for accuracy. The localization error (LE) was subsequently measured in the planning software as the Euclidean distance between the center of the phantom FM and the center of the probe FM.

\section{Retrospective Clinical Study}

We started to use the Neurolocate module systematically in the fall of 2016 at our center, following a period 
of preliminary optimization and validation. For the retrospective analysis, we therefore selected a series of 8 consecutive patients who underwent SEEG implantations between September and December 2016.

LE was measured in the planning software as the Euclidean distance between the planned cortical entry point and the major axis of the electrode (entry point LE) and between the planned target point and the tip of the implanted electrode (target point LE). The measurement procedure was detailed in previous publications. ${ }^{4,6}$

Data from the 8 selected patients were compared with data from a historical control group. ${ }^{4}$

We intended to check whether the accuracy of a new technique, frameless registration with the Neurolocate module, was noninferior to frame-based registration with a Talairach frame, which was considered as the gold standard. In fact, we recently reported 237 SEEG procedures with 3252 electrodes that caused no major intracranial bleeding. ${ }^{2}$ It was necessary to define which is the higher LE still acceptable for SEEG safety, to estimate the confidence interval. The group at Fondation Rothschild, Paris, has, over a long period, used the Neuromate stereotactic robot in frameless conditions with an ultrasound (US) device for patient registration ${ }^{9-11}$ and reported no major bleeding; thus, we obtained useful data from a previous phantom study comparing Neuromate accuracy in framebased and US-frameless conditions..$^{15}$ The authors reported mean LEs $( \pm \mathrm{SD})$ in frame-based and US-frameless conditions of $0.86 \pm 0.32 \mathrm{~mm}$ and $1.95 \pm 0.44 \mathrm{~mm}$, respectively. Based on these data, we designed a 2-arm study with a minimum sample size equal to 12 patients in each arm (total sample size 24), reaching a power greater than 99\% to detect the specified noninferiority, at a significance level of $\alpha=0.025$, accordingly to the suggestions given by Chow and coauthors. ${ }^{8}$

We largely respected the minimum requirements of the study design, since we tested 40 trajectories (20 for each arm of the study).

\section{Statistical Analysis}

Normality of values was tested with the Shapiro-Wilk test. Mean values were compared with the Welch 2-sample t-test, if the data were normally distributed; for data that were not normally distributed, median values were compared with the Kruskal-Wallis test. Since the phantom study was a noninferiority one, values of $p<0.025$ were considered as evidence of findings not attributable to chance. For the retrospective clinical comparison, however, the significance threshold was set at 0.05 . Statistical analysis was performed with R 3.3, developed by the R Foundation for Statistical Computing (https://www.Rproject.org/).

\section{Approvals}

The Niguarda Hospital ethics committee approved both studies.

\section{Results \\ Phantom Study}

The raw data from the phantom study are reported in
Table 1 . The LE values were normally distributed with both Neurolocate and frame-based registration.

The mean LE was $0.67 \pm 0.29 \mathrm{~mm}$ for Neurolocatebased trajectories and $0.76 \pm 0.34 \mathrm{~mm}$ for frame-based trajectories $(p=0.35)$, indicating that the accuracy of Neurolocate-based registration is noninferior to that of frame-based registration. The LE values for the Neurolocate-based and frame-based trajectories ranged from 0.34 to $1.37 \mathrm{~mm}$ and from 0.16 to $1.54 \mathrm{~mm}$, respectively.

\section{Retrospective Clinical Study}

Eight consecutive patients ( 5 male, 3 female) underwent implantation of a total of 127 intracerebral SEEG electrodes with a Neurolocate-registration-based procedure. The historical control group was made up of 78 patients (49 male, 29 female) who underwent 81 SEEG electrode implantation procedures with a frame-registration-based procedure, for positioning of a total of 1050 intracerebral electrodes. ${ }^{4}$ The mean age of the patients was $25.5 \pm 10.4$ years in the group that underwent Neurolocate-registration-based procedures and $25 \pm 11$ years in the group that underwent frame-registration-based procedures $(\mathrm{p}=0.9)$.

In 7 of the 8 Neurolocate-registration-based procedures, the patient's head was fixed with the Talairach frame, used only as a head holder. In the remaining procedure, the patient's head was fixed with a Sugita-like headrest system. All of the SEEG procedures were successfully completed, and no complications occurred.

The entry point LEs and target point LEs were not normally distributed. The median entry point LE was 0.59 mm (IQR 0.25-0.88) for Neurolocate-registration-based trajectories and $0.78 \mathrm{~mm}$ (IQR 0.49-1.08) for frame-registration-based trajectories $(\mathrm{p}=0.00002)$. The median target point LE was $1.49 \mathrm{~mm}$ (IQR 1.06-2.4) for Neurolocate-registration-based and $1.77 \mathrm{~mm}$ (IQR 1.25-2.5) for frame-registration-based trajectories $(\mathrm{p}=0.05)$.

\section{Discussion}

To the best of our knowledge, this is the first study reporting on the accuracy of Neurolocate-based registration with the Neuromate sterotactic robot.

No major bleedings occurred in the previously reported 237 SEEG procedures (3252 electrodes) performed with the frame-based workflow used at our center since fall 2008. ${ }^{2}$ Since we demonstrated that Neurolocate registration is not inferior to the gold standard, it should have a safety profile similar to frame-based registration.

\section{Accuracy in Phantom Studies for Robotic Brain Surgery}

Our 40-trajectory phantom study demonstrated that the stereotactic accuracy obtained with Neurolocate registration is not inferior to that obtained in frame-based conditions.

Of note, it must be considered that with our method the final LE is the sum of 4 components: 1) the geometrical characteristics of the planning image data set, 2) the registration error, 3) the intrinsic mechanical error of the robotic arm, and 4) the measurement error. The intrinsic accuracy of the Neuromate robot, measured at the time of the phantom study, was $0.45 \mathrm{~mm}$. Thus, the part of the LE 
TABLE 1. Results of phantom study

\begin{tabular}{|c|c|c|c|c|c|c|c|c|}
\hline \multirow[b]{2}{*}{ Trajectory } & \multirow[b]{2}{*}{ Exp No. } & \multirow[b]{2}{*}{ LE } & \multicolumn{3}{|c|}{ Target Coordinates (mm) } & \multicolumn{3}{|c|}{ Entry Coordinates (mm) } \\
\hline & & & $\mathrm{X}$ & Y & Z & $x$ & Y & Z \\
\hline Exp1_Neurolocate_Target01 & 1 & 0.41 & 109.5 & 64.4 & 73.2 & 124.3 & 64.4 & 36.8 \\
\hline Exp1_Neurolocate_Target02 & 1 & 0.35 & 141.6 & 55.0 & 56.9 & 138.5 & 94.3 & 12.0 \\
\hline Exp1_Neurolocate_Target03 & 1 & 0.54 & 146.7 & 87.6 & 71.8 & 146.7 & 85.7 & 1.2 \\
\hline Exp1_Neurolocate_Target04 & 1 & 0.51 & 154.1 & 108.5 & 69.5 & 154.2 & 110.2 & 2.1 \\
\hline Exp1_Neurolocate_Target05 & 1 & 0.34 & 145.6 & 142.7 & 77.7 & 145.6 & 143.8 & 5.5 \\
\hline Exp1_Neurolocate_Target06 & 1 & 0.66 & 80.6 & 151.0 & 80.0 & 78.9 & 151.0 & 2.1 \\
\hline Exp1_Neurolocate_Target07 & 1 & 0.36 & 56.6 & 111.8 & 81.3 & 56.6 & 110.2 & 5.9 \\
\hline Exp1_Neurolocate_Target08 & 1 & 0.53 & 71.7 & 85.4 & 84.2 & 71.7 & 84.7 & 2.1 \\
\hline Exp1_Neurolocate_Target09 & 1 & 0.56 & 78.7 & 62.5 & 56.7 & 78.7 & 61.8 & 3.6 \\
\hline Exp1_Neurolocate_Target10 & 1 & 0.69 & 104.6 & 49.2 & 70.8 & 104.6 & 45.4 & 2.9 \\
\hline Exp1_Frame_Target01 & 1 & 1.08 & 109.5 & 64.4 & 73.2 & 124.3 & 64.4 & 36.8 \\
\hline Exp1_Frame_Target02 & 1 & 1.54 & 141.6 & 55.0 & 56.9 & 138.5 & 94.3 & 12.0 \\
\hline Exp1_Frame_Target03 & 1 & 0.60 & 146.7 & 87.6 & 71.8 & 146.7 & 85.7 & 1.2 \\
\hline Exp1_Frame_Target04 & 1 & 0.53 & 154.1 & 108.5 & 69.5 & 154.2 & 110.2 & 2.1 \\
\hline Exp1_Frame_Target05 & 1 & 0.33 & 145.6 & 142.7 & 77.7 & 145.6 & 143.8 & 5.5 \\
\hline Exp1_Frame_Target06 & 1 & 0.73 & 80.6 & 151.0 & 80.0 & 78.9 & 151.0 & 2.1 \\
\hline Exp1_Frame_Target07 & 1 & 0.16 & 56.6 & 111.8 & 81.3 & 56.6 & 110.2 & 5.9 \\
\hline Exp1_Frame_Target08 & 1 & 1.21 & 71.8 & 85.6 & 84.1 & 71.7 & 84.7 & 2.1 \\
\hline Exp1_Frame_Target09 & 1 & 0.73 & 78.7 & 62.5 & 56.7 & 78.7 & 61.8 & 3.6 \\
\hline Exp1_Frame_Target10 & 1 & 0.90 & 104.6 & 49.2 & 70.8 & 104.6 & 45.4 & 2.9 \\
\hline Exp2_Neurolocate_Target01 & 2 & 0.99 & 104 & 66.7 & 77.7 & 104 & 71.7 & 5.5 \\
\hline Exp2_Neurolocate_Target02 & 2 & 0.79 & 136 & 52.2 & 65.7 & 136 & 64 & 4.7 \\
\hline Exp2_Neurolocate_Target03 & 2 & 0.64 & 143.6 & 85.7 & 76.9 & 143.6 & 87.3 & 5.5 \\
\hline Exp2_Neurolocate_Target04 & 2 & 0.38 & 153.5 & 105.2 & 72.9 & 153.5 & 105.3 & 5.9 \\
\hline Exp2_Neurolocate_Target05 & 2 & 0.42 & 148.4 & 141.0 & 76.7 & 148.4 & 136.2 & 5.2 \\
\hline Exp2_Neurolocate_Target06 & 2 & 0.75 & 84.8 & 156.2 & 72.1 & 84.8 & 151.4 & 3.6 \\
\hline Exp2_Neurolocate_Target07 & 2 & 0.83 & 56.8 & 120.3 & 75.6 & 56.8 & 129.3 & 4.8 \\
\hline Exp2_Neurolocate_Target08 & 2 & 1.37 & 68.4 & 92.9 & 82.5 & 68.4 & 104.5 & 7.5 \\
\hline Exp2_Neurolocate_Target09 & 2 & 1.17 & 74.8 & 66.2 & 58.7 & 74.8 & 71.7 & 4.8 \\
\hline Exp2_Neurolocate_Target10 & 2 & 1.05 & 97.6 & 52.1 & 76.8 & 97.6 & 61.8 & 5.5 \\
\hline Exp2_Frame_Target01 & 2 & 0.77 & 104 & 66.7 & 77.7 & 104 & 71.7 & 5.5 \\
\hline Exp2_Frame_Target02 & 2 & 1.03 & 136 & 52.2 & 65.7 & 136 & 64 & 4.7 \\
\hline Exp2_Frame_Target03 & 2 & 0.69 & 143.6 & 85.7 & 76.9 & 143.6 & 87.3 & 5.5 \\
\hline Exp2_Frame_Target04 & 2 & 1.00 & 153.5 & 105.2 & 72.9 & 153.5 & 105.3 & 5.9 \\
\hline Exp2_Frame_Target05 & 2 & 0.47 & 148.4 & 141.0 & 76.7 & 148.4 & 136.2 & 5.2 \\
\hline Exp2_Frame_Target06 & 2 & 0.46 & 84.8 & 156.2 & 72.1 & 84.8 & 151.4 & 3.6 \\
\hline Exp2_Frame_Target07 & 2 & 0.33 & 56.8 & 120.3 & 75.6 & 56.8 & 129.3 & 4.8 \\
\hline Exp2_Frame_Target08 & 2 & 1.00 & 68.4 & 92.9 & 82.5 & 68.4 & 104.5 & 7.5 \\
\hline Exp2_Frame_Target09 & 2 & 0.72 & 74.8 & 66.2 & 58.7 & 74.8 & 71.7 & 4.8 \\
\hline Exp2_Frame_Target10 & 2 & 0.95 & 97.6 & 52.1 & 76.8 & 97.6 & 61.8 & 5.5 \\
\hline
\end{tabular}

Exp $=$ experiment.

caused by the Neurolocate module is only a few tenths of a millimeter.

Li et al. ${ }^{15}$ compared the accuracy of Neuromate in frame-based and US-frameless conditions, reporting mean $( \pm \mathrm{SD})$ LEs of $0.86 \pm 0.32 \mathrm{~mm}$ and $1.95 \pm 0.44 \mathrm{~mm}$, respectively. Our results in frame conditions are similar, while our frameless ones are definitely better. Thus, we conclude that the Neurolocate system is the best option for frameless registrations with the Neuromate stereotactic robot.

Varma and Eldridge ${ }^{26}$ reported results from a study performed with Neuromate and US-frameless registration, too. The mean LE was $1.29 \mathrm{~mm}$, taking into account that the slice thickness of the CT planning data set was 1.5 $\mathrm{mm}$, under their best experimental conditions.

Lefranc et al. ${ }^{14}$ reported a mean accuracy $( \pm \mathrm{SD})$ of 0.3 
$\pm 0 \mathrm{~mm}$ with identical $\mathrm{O}$-arm image data sets, frameless laser-based registration, and the ROSA robotic system (Medtech) in a 20-trajectory study. Nevertheless, it is difficult to compare our results with theirs because they did not detail the measurement method. We measured the LE in the image space, likely overestimating it. However, our method is the only one that can be adopted in both phantom and clinical conditions, and therefore it is advantageous to make a comparison between in vitro and in vivo conditions.

Minchev et al. ${ }^{18}$ reported a mean accuracy of $0.6 \mathrm{~mm}$ (range $0.1-0.9 \mathrm{~mm}$ ) with a 3D CT data set (slice thickness $0.625 \mathrm{~mm}$ ), optical tracking registration (Medtronic StealthStation S7), and iSYS1 robot (Medizintechnik) in a 162-trajectory study. LEs were measured by means of a digital caliper. The same group reported a mean accuracy $( \pm \mathrm{SD})$ of $0.8 \pm 0.7 \mathrm{~mm}$, based on 5 trajectories, in another study. The analysis was carried out in similar conditions, adding some hardware for SEEG procedure optimization. ${ }^{10}$

Von Langsdorff et al. ${ }^{27}$ reported a mean accuracy $( \pm$ SD) of $0.44 \pm 0.23 \mathrm{~mm}$, coupling the Fisher frame and Neuromate in a 21-trajectory study. Nevertheless, details about the planning data sets and the coregistration method were not provided.

In conclusion, the above-listed phantom studies suggest that US-frameless registration is less accurate than other methods. Other registration techniques and devices provided similar results, but the heterogeneity of study methods make a rigorous comparison difficult.

\section{Accuracy in Clinical Studies for SEEG}

SEEG-derived accuracy estimation is intrinsically different from deep brain stimulation and biopsy procedures, under clinical conditions. In fact, SEEG electrodes are semirigid and implanted without any guide tubes, so intracranial deviations can occur. Therefore, the entry point LE is the best measure to evaluate the accuracy of the stereotactic system in SEEG implantations. The cortical entry point is also the most important region for safety profiles. ${ }^{4}$

Gonzalez-Martinez et al. ${ }^{12}$ reported a median entry point LE of $1.2 \mathrm{~mm}$ (IQR $0.78-1.83 \mathrm{~mm}$ ) from 500 electrodes implanted with the ROSA robot in frameless conditions.

Dorfer et al. ${ }^{10}$ reported a mean entry point LE of 1.3 $\mathrm{mm}$ (range 0.1-3.4 mm) from 93 electrodes implanted with optical tracking registration and an iSYS1 robot. For the subset of the most recent 31 electrode implantations with optimized hardware, they reported a mean entry point LE ( \pm SD) of $1.18 \pm 0.5 \mathrm{~mm}$.

Roessler et al., ${ }^{23}$ reported a mean entry point LE ( \pm SD) of $1.4 \pm 1.2 \mathrm{~mm}$ from 58 electrodes implanted with the use of an intraoperative MR scanner (Magnetom Sonata; Siemens Medical Solutions) and an optical-tracking-based navigation system (Brainlab AG).

In summary, all of the previously reported entry point LEs are larger than $1 \mathrm{~mm}$ in real clinical conditions, while our results with Neuromate have been submillimetric with both frame-based and Neurolocate registration methods. This suggests that use of the Neurolocate system could further reduce the rate of intracranial bleedings, although our clinical results are based on only 127 trajectories from 8 patients. $^{2}$

Our experience with the Neurolocate system is limited. Nevertheless, it is a promising tool that provides an accurate and easy registration with a compatible intraoperative CT scanner. We do not need to take the patient to an external CT room, thanks to the availability of the intraoperative imaging system.

In the future, we will collect further data to assess whether Neurolocate registration can effectively reduce SEEG surgical time.

\section{Conclusions}

The Neurolocate patient registration module is a brand new, frameless, noninvasive registration tool compatible with Neuromate robotic assistant. Its use can increase the ease of modern robotic SEEG procedures, while still having an accuracy and safety profile similar to that of framebased registration.

\section{Acknowledgments}

We thank Renishaw Mayfield for providing us with the measurement probe.

\section{References}

1. Abhinav K, Prakash S, Sandeman DR: Use of robot-guided stereotactic placement of intracerebral electrodes for investigation of focal epilepsy: initial experience in the UK. Br J Neurosurg 27:704-705, 2013

2. Cardinale F: Stereoelectroencephalography: application accuracy, efficacy, and safety. World Neurosurg 94:570-571, 2016

3. Cardinale F, Casaceli G, Raneri F, Miller J, Lo Russo G: Implantation of stereoelectroencephalography electrodes: a systematic review. J Clin Neurophysiol 33:490-502, 2016

4. Cardinale F, Cossu M, Castana L, Casaceli G, Schiariti MP, Miserocchi A, et al: Stereoelectroencephalography: surgical methodology, safety, and stereotactic application accuracy in 500 procedures. Neurosurgery 72:353-366, 2013

5. Cardinale F, González-Martínez J, Lo Russo G: SEEG, Happy Anniversary! World Neurosurg 85:1-2, 2016 (Letter)

6. Cardinale F, Miserocchi A, Moscato A, Cossu M, Castana L, Schiariti MP, et al: Talairach methodology in the multimodal imaging and robotics era, in Scarabin JM (ed): Stereotaxy and Epilepsy Surgery. Montrouge, France: John Libbey Eurotext, 2012, pp 245-272

7. Cardinale F, Pero G, Quilici L, Piano M, Colombo P, Moscato A, et al: Cerebral angiography for multimodal surgical planning in epilepsy surgery: description of a new threedimensional technique and literature review. World Neurosurg 84:358-367, 2015

8. Chow SC, Wang H, Shao J (eds): Sample Size Calculations in Clinical Research, ed 2. New York: Chapman and Hall/ CRC, 2008

9. Delalande O, Dorfmüller G, Ferrand-Sorbets S: Epilepsy surgery in children, in Scarabin JM (ed): Stereotaxy and Epilepsy Surgery. Montrouge, France: John Libbey Eurotext, 2012, pp 329-339

10. Dorfer C, Minchev G, Czech T, Stefanits H, Feucht M, Pataraia $\mathrm{E}$, et al: A novel miniature robotic device for frameless implantation of depth electrodes in refractory epilepsy. J Neurosurg [epub ahead of print August 5, 2016. DOI: 10.3171/2016.5.JNS16388]

11. Dorfmüller G, Ferrand-Sorbets S, Fohlen M, Bulteau C, 
Archambaud F, Delalande O, et al: Outcome of surgery in children with focal cortical dysplasia younger than 5 years explored by stereo-electroencephalography. Childs Nerv Syst 30:1875-1883, 2014

12. González-Martínez J, Bulacio J, Thompson S, Gale J, Smithason S, Najm I, et al: Technique, results, and complications related to robot-assisted stereoelectroencephalography. Neurosurgery 78:169-180, 2016

13. Jayakar P, Gotman J, Harvey AS, Palmini A, Tassi L, Schomer D, et al: Diagnostic utility of invasive EEG for epilepsy surgery: Indications, modalities, and techniques. Epilepsia 57:1735-1747, 2016

14. Lefranc M, Capel C, Pruvot AS, Fichten A, Desenclos C, Toussaint $\mathrm{P}$, et al: The impact of the reference imaging modality, registration method and intraoperative flat-panel computed tomography on the accuracy of the ROSA ${ }^{\circledR}$ stereotactic robot. Stereotact Funct Neurosurg 92:242-250, 2014

15. Li QH, Zamorano L, Pandya A, Perez R, Gong J, Diaz F: The application accuracy of the NeuroMate robot--A quantitative comparison with frameless and frame-based surgical localization systems. Comput Aided Surg 7:90-98, 2002

16. Lo Russo G, Tassi L, Cossu M, Cardinale F, Mai R, Castana $\mathrm{L}$, et al: Focal cortical resection in malformations of cortical development. Epileptic Disord 5 (Suppl 2):S115-S123, 2003

17. McGonigal A, Bartolomei F, Régis J, Guye M, Gavaret M, Trébuchon-Da Fonseca A, et al: Stereoelectroencephalography in presurgical assessment of MRI-negative epilepsy. Brain 130:3169-3183, 2007

18. Minchev G, Kronreif G, Martínez-Moreno M, Dorfer C, Micko A, Mert A, et al: A novel miniature robotic guidance device for stereotactic neurosurgical interventions: preliminary experience with the iSYS1 robot. J Neurosurg 126:985-996, 2017

19. Moshé SL, Perucca E, Ryvlin P, Tomson T: Epilepsy: new advances. Lancet 385:884-898, 2015

20. Mullin JP, Shriver M, Alomar S, Najm I, Bulacio J, Chauvel $\mathrm{P}$, et al: Is SEEG safe? A systematic review and meta-analysis of stereo-electroencephalography-related complications. Epilepsia 57:386-401, 2016

21. Munari C, Bancaud J: The role of stereo-electroencephalography (SEEG) in the evaluation of partial epileptic seizures, in Porter RJ, Morselli PL (eds): The Epilepsies. Bodmin, UK: Butterworth \& Co., 1985, pp 267-306

22. Munari C, Lo Russo G, Minotti L, Cardinale F, Tassi L, Kahane P, et al: Presurgical strategies and epilepsy surgery in children: comparison of literature and personal experiences. Childs Nerv Syst 15:149-157, 1999
23. Roessler K, Sommer B, Merkel A, Rampp S, Gollwitzer S, Hamer HM, et al: A frameless stereotactic implantation technique for depth electrodes in refractory epilepsy utilizing intraoperative MR imaging. World Neurosurg 94:206-210, 2016

24. Schuele SU: Stereoelectroencephalography. J Clin Neurophysiol 33:477, 2016

25. Serletis D, Bulacio J, Bingaman W, Najm I, GonzálezMartínez J: The stereotactic approach for mapping epileptic networks: a prospective study of 200 patients. J Neurosurg 121:1239-1246, 2014

26. Varma TRK, Eldridge P: Use of the NeuroMate stereotactic robot in a frameless mode for functional neurosurgery. Int $\mathbf{J}$ Med Robot 2:107-113, 2006

27. von Langsdorff $\mathrm{D}$, Paquis $\mathrm{P}$, Fontaine $\mathrm{D}$ : In vivo measurement of the frame-based application accuracy of the neuromate neurosurgical robot. J Neurosurg 122:191-194, 2015

28. Wiebe S, Blume WT, Girvin JP, Eliasziw M: A randomized, controlled trial of surgery for temporal-lobe epilepsy. N Engl J Med 345:311-318, 2001

\section{Disclosures}

Francesco Cardinale reports a consultant relationship (paid) with Renishaw Mayfield, the manufacturer of the Neuromate robotic system. He also reports that he has previously served as a consultant (advisory board member) for Medtronic.

\section{Author Contributions}

Conception and design: Cardinale, De Momi, Castana. Acquisition of data: Rizzi, Cardinale. Analysis and interpretation of data: Cardinale. Drafting the article: Rizzi, Cardinale. Critically revising the article: Rizzi, Cardinale, Castana. Reviewed submitted version of manuscript: Rizzi, Cardinale, d'Orio, Casaceli, Arnulfo, Narizzano, Scorza, Redaelli, Sberna, Moscato, Castana. Approved the final version of the manuscript on behalf of all authors: Rizzi. Statistical analysis: Cardinale, Nichelatti, Sberna, Moscato. Administrative/technical/material support: d'Orio, Casaceli, Arnulfo, Narizzano, Scorza, De Momi, Nichelatti, Redaelli. Study supervision: Cardinale, d'Orio, Casaceli, Arnulfo, Narizzano, Scorza, De Momi, Redaelli, Sberna, Moscato, Castana.

\section{Correspondence}

Michele Rizzi, "Claudio Munari” Center for Epilepsy Surgery, Niguarda Hospital, Piazza Ospedale Maggiore 3, Milan 20162, Italy. email: michele.rizzi@ospedaleniguarda.it. 